\title{
Establishment of a nasopharyngeal carcinoma cell line capable of undergoing lytic Epstein-Barr virus reactivation
}

\author{
Yim Ling Yip ${ }^{1} \cdot$ Weitao Lin ${ }^{1} \cdot$ Wen Deng ${ }^{2} \cdot$ Lin Jia $^{1} \cdot$ Kwok Wai Lo ${ }^{3} \cdot$ Pierre Busson $\bigodot^{4} \cdot$ Benjamin Vérillaud ${ }^{4}$. \\ Xuefeng Liu $\mathbb{B}^{5,6} \cdot$ Chi Man Tsang ${ }^{1} \cdot$ Maria Li Lung ${ }^{7}$ Sai Wah Tsao ${ }^{1}$
}

Received: 11 August 2017 / Revised: 22 December 2017 / Accepted: 10 January 2018 / Published online: 16 May 2018

(c) United States \& Canadian Academy of Pathology 2018

\begin{abstract}
Epstein-Barr virus (EBV) infects more than $90 \%$ of the adult human population. Undifferentiated nasopharyngeal carcinoma (NPC) is common in Southeast Asia, with a particularly high incidence among southern Chinese. The EBV genome can be detected in practically all cancer cells in undifferentiated NPC. The role of EBV in pathogenesis of undifferentiated NPC remains elusive. NPC cell lines are known to be difficult to establish in culture. The EBV+ve NPC cell lines, even if established in culture, rapidly lost their EBV episomes upon prolonged propagation. At present, the C666-1 NPC cell line, which is defective in lytic EBV reactivation, is the only EBV+ve NPC cell line available for NPC and EBV research. The need to establish new and representative NPC cell lines is eminent for NPC and EBV research. In this study, we report the use of the Rho-associated kinase inhibitor (Y-27632) has facilitated the establishment of a new EBV+ve NPC cell line from an earlier established NPC xenograft, C17. The C17 cell line was tumorigenic in immune-deficient mice (NOD/SCID). It retained the EBV episomes and could be induced to undergo productive lytic reactivation of EBV to generate infectious virus particles. The $\mathrm{C} 17$ cell line represents a new investigative tool for NPC and EBV studies. The ability of C17 to undergo lytic reactivation is unique and opens up the opportunity to examine regulation of latent and lytic infection of EBV and their contributions to NPC pathogenesis.
\end{abstract}

These authors contributed equally: Yim Ling Yip, Weitao Lin.

Electronic supplementary material The online version of this article (https://doi.org/10.1038/s41374-018-0034-7) contains supplementary material, which is available to authorized users.

$\triangle$ Sai Wah Tsao

gswtsao@hku.hk

1 School of Biomedical Sciences, Li Ka Shing Faculty of Medicine, University of Hong Kong, Hong Kong SAR, China

2 School of Nursing, Li Ka Shing Faculty of Medicine, University of Hong Kong, Hong Kong SAR, China

3 Department of Anatomical and Cellular Pathology, State Key Laboratory in Oncology in South China, Prince of Wales Hospital, Shatin, Hong Kong

4 Laboratoire de Biologie des Tumeurs Humaines, Institut Gustave Roussy, Villejuif, France

5 Department of Pathology and Center for Cellular Reprogramming, Georgetown University Medical Center, Washington, DC, USA

6 Cancer Hospital, Guangzhou Medical University, Guangzhou, Guangdong, China

7 Department of Clinical Oncology, Li Ka Shing Faculty of Medicine, University of Hong Kong, Hong Kong SAR, China

\section{Introduction}

Undifferentiated nasopharyngeal carcinoma (NPC) is highly prevalent among southern Chinese, particularly the ethnic Cantonese population. One of the unique features of undifferentiated NPC is the universal presence of Epstein-Barr virus (EBV) infection. EBV infection is believed to be an early event in NPC development and plays an important role in its pathogenesis. Expression of latent EBV genes, including EBER1/2, EBNA1, LMP1, LMP2, and BART-microRNAs, are detected in primary NPC and may contribute to pathogenesis as well as progression of NPC [1].

A major obstacle in the field of NPC and EBV studies is the scarcity of representative NPC cell lines for investigations. Most of the commonly used NPC cell lines are contaminated with obscure genetic elements of HeLa cells $[2,3]$. Furthermore, all except one of the previously reported NPC cell lines lost their EBV episomes upon prolonged subculture (within 10-20 passages), which limits their use as representative NPC cell lines. The C666-1 is the only EBV+ve NPC cell line used extensively in NPC and 
EBV research. It was established from an earlier established NPC xenograft (xeno-666), which has been passaged in nude mice [4]. The C666-1 is defective in lytic reactivation of EBV, which may account for its ability to retain EBV episomes upon continuous propagation in culture. EBV infection is an essential feature of NPC. Representative NPC cell lines harboring EBV episomes are important investigative tools in understanding the contribution of EBV infection in NPC pathogenesis and progression.

For unclear reasons, establishment of cell lines in culture from NPC is known to be difficult. The rich inflammatory tumor microenvironment in NPC probably plays an essential role in providing the growth factors and stromal cell interaction essential to support growth of NPC cells in patients as well as maintenance of latent EBV infection in NPC cells. Furthermore, the undifferentiated property of NPC cells may also be crucial for the maintenance of EBV episomes. Explantation of NPC tissues in culture medium lacking these supportive growth factors and stromal cell interaction may alter the differentiation properties of NPC cells, rendering them non-permissive to retain EBV. This contrasts greatly with EBV infection of B cells, which confers proliferation advantages to infected B cells. Our experience with establishment of NPC cells from biopsies and surgical specimens was that epithelial outgrowths from NPC specimens readily stopped proliferation suggestive of suboptimal culture conditions to sustain the growth of EBVinfected NPC cells. The EBV+ve C666-1 was established from the xenograft, X666, which has been passaged 18 times in nude mice and may be less dependent on the supportive stromal factors for growth [4]. A previous study also showed that cell line establishment from xenografted tissues is much more efficient ( $47 \%$ success rate) compared to that from fresh tumor specimens (9.7\% success rate) [5]. We have previously attempted to establish NPC cell lines from established NPC xenografts, including xeno-2117, $\mathrm{C} 15$, and $\mathrm{C} 17$ using conventional culture medium composed of RPMI 1640 supplemented with $10 \%$ fetal bovine serum but failed (unpublished observations). Recent studies have reported that inhibition of the Rho-associated kinase (ROCK) by Y-27632 induces a stem-like phenotype and reprogramming of embryonic stem cells [6] and epithelial cells $[7,8]$. Treatment of cells with ROCK inhibitor could prevent growth arrest, inhibit terminal differentiation of squamous epithelial cells, support cell proliferation, and immortalize keratinocyte [9-11]. These special properties of ROCK inhibitor may be advantageous for the establishment of $\mathrm{EBV}+\mathrm{ve}$ NPC cell lines, which are predominantly undifferentiated in nature. Furthermore, we have reported stemness properties in the C666-1 cells [12], which may be crucial for its ability to retain EBV episomes. In this study, we have applied the use of spheroid cultures in the presence of ROCK inhibitor (Y-27632) and successfully established a new $\mathrm{EBV}+$ ve NPC cell line from the C17 NPC xenograft. Interestingly, the newly established $\mathrm{C} 17$ cell line could be induced to undergo lytic reactivation upon expression of BZLF1 (Zta) which is the key viral gene regulating the latent to lytic switch of EBV infection. The $\mathrm{C} 17$ represents the first NPC cell line which is amenable to lytic reactivation to produce infectious EBV particles.

\section{Materials and methods}

\section{Xenograft and cell lines}

The C17 NPC xenograft used to establish the EBV+ve NPC cell line reported in this study was originally established in athymic nude mice from a cutaneous metastasis from the late stage of a male patient with poorly differentiated $\mathrm{EBV}+\mathrm{ve}$ NPC admitted at the Institut Gustave Roussy [13, 14]. The patient had received multiple treatment. Oral informed consent was obtained from the patient, which was in accordance with the laws in force at that time. For 20 years, passages were performed each month on Swiss nude mice bread in animal facility in Institut Gustave Roussy with the same protocol. Tumors from donor mice were minced in fragments of $1-3 \mathrm{~mm}$ in diameter. The most necrotic fragments (white) were discarded. About $1.5 \mathrm{mg}$ of wet fragments were implanted subcutaneously in recipient mice. The xenograft was passaged on BALB/cAnN-nu (nude) mice in laboratory animal unit in the University of Hong Kong. Procedures for mouse experiment were approved by the animal experimentation ethics committee of the Institut Gustave Roussy (CEEA-26 - decision \#2012079) and the Committee on the Use of Live Animals in Teaching and Research in the University of Hong Kong. Establishment of the $\mathrm{C} 17$ cell line was initiated from the explant culture of C17 xenografted tissues in 2012, after 4 passages in the University of Hong Kong. To initiate the epithelial outgrowth from the C17 NPC xenograft, the xenografted tumor was cut into small pieces around $1 \mathrm{~mm}^{3}$ in sizes and explanted to a T-25 culture flask (Thermo Fisher Scientific, Waltham, MA) initially with $2 \mathrm{ml}$ of RPMI 1640 medium (Sigma, St. Louis, MO) supplemented with $10 \%$ fetal bovine serum (GIBCO, Thermo Fisher Scientific), $100 \mathrm{U} / \mathrm{ml}$ penicillin and $100 \mu \mathrm{g} / \mathrm{ml}$ streptomycin. In addition, $4 \mu \mathrm{M}$ of ROCK inhibitor, Y-27632 (Enzo Life Sciences, Inc., Farmingdale, NY), was included in the culture medium. Details of the establishment of the C17 as continuous cell line in culture are described in "Results". The Namalwa, C666-1, and EBV-uninfected Akata cells were cultured in RPMI 1640 medium supplemented with $10 \%$ fetal bovine serum, $100 \mathrm{U} / \mathrm{ml}$ penicillin, and $100 \mu \mathrm{g} / \mathrm{ml}$ streptomycin. All the cell cultures were maintained in a $37^{\circ} \mathrm{C}$ incubator with $5 \% \mathrm{CO}_{2}$ in air. 


\section{Spectral karyotyping (SKY) analysis}

The methods for metaphase chromosome spreads and spectral karyotyping have been published previously [15]. Metaphase preparations of the $\mathrm{C} 17$ cells were stained with 24-color SKYPaint probe (Applied Spectral Imaging, Migdal, Ha'Emek, Israel) and image detection was carried out according to the recommendations from manufacturer. SKY images were captured using the SkyVision Imaging System equipped with a Zeiss Axioplan 2 fluorescence microscope. Karyotyping was performed using SKY View 2.0 software (Applied Spectral Imaging).

\section{Soft agar growth assay}

Anchorage-independent growth ability of the established C17 cell line was determined by soft agar assay. The assay was carried out in a 6-well plate (Greiner Bio-One, Monroe, NC) with $0.6 \%$ bacto-agar base and $0.3 \%$ bacto-agar top containing $5 \times 10^{4}$ cells. Anchorage-independent colonies were counted 2 months after incubation. Colonies greater than $0.2 \mathrm{~mm}$ in diameter, containing more than $50-60$ cells, were counted [16].

\section{Tumorigenesis in mice}

The tumorigenicity of the established $\mathrm{C} 17$ cell line was investigated by subcutaneous injection in NOD/SCID mice. About $1 \times 10^{7}$ cells $(100 \mu \mathrm{l})$ were mixed with equal volume of Matrigel (BD Biosciences, San Jose, CA) and injected subcutaneously into each of the 4- to 6-week-old male NOD/SCID mice. Six mice were injected and the growth of tumor was measured every 3-4 days until the tumor was around $2 \mathrm{~cm}^{3}$ in size.

\section{Determination of the EBV genome copy number by quantitative PCR}

DNA from the established $\mathrm{C} 17$ cell line and its parental $\mathrm{C} 17$ xenograft was extracted using DNeasy blood and tissue kit (Qiagen, Venlo, the Netherlands) according to the manufacturer's instructions. PCR amplification was carried out on MyiQ2 Two Color Real-Time PCR detection system (BioRad, Hercules, CA) using LightCycler 480 Probe Master with specific probe from Universal Probe Library Set, Human (Roche Applied Science, Penzberg, Upper Bavaria, Germany). The primers and probes for human $\beta$ globin and EBNA1 are listed in Supplementary Table 1. The expression level of EBNAI transcript was internally normalized using the expression level of human $\beta$-globin. The relative expression of EBNA1 was determined by the $2^{-\Delta \Delta \mathrm{Ct}}$ method. The absolute EBV genome copy per cell was estimated after normalization to the EBV DNA in Namalwa which contains 2 copies of integrated EBV genome per cell [17].

\section{EBV gene expression profile by RT-PCR analysis}

Extraction of total RNA and the reverse transcription to cDNA was performed as previously described [18]. Expression levels of EBV genes were examined by quantitative RT-PCR using the PCR machine and reagents as mentioned above. The primer sequences with specific probes are listed in Supplementary Table 1 . The expression levels of the EBV genes were normalized with human glyceraldehyde 3-phosphate dehydrogenase (GAPDH). The relative expression level of genes of interest was determined by the $2^{-\Delta \Delta C t}$ method.

\section{Western blot analysis}

The Western blot analysis was carried out as previously described [19]. Antibodies against Zta and Rta were purchased from Argene (bioMérieux SA, France). An antibody against EA-D was kindly provided by Prof. Jaap Middeldorp (VU University Medical Center, the Netherlands). The antibody against $\beta$-actin was from Santa Cruz Biotechnology (Dallas, TX).

\section{Fluorescence in situ hybridization (FISH) for the EBV genome}

In this study, EBV FISH was also performed to examine the presence of EBV genomes using a DNA probe targeting BamHI-W repeats of the EBV genome. The biotin-labeled EBV probe was kindly provided by Prof. Bill Sugden (McArdle Laboratory for Cancer Research, University of Wisconsin-Madison, Madison, WI, USA). The detailed procedures have been published previously [20].

\section{Histology and EBER in situ hybridization}

Paraffin sections of $5 \mu \mathrm{m}$ thickness were prepared from the embedded tissues. The paraffin sections were de-waxed and rehydrated before used for hematoxylin \& eosin (H\&E) staining for histopathological examination and for EBER in situ hybridization (ISH) to detect the presence of EBV. The procedures for EBV ISH using EBV probe ISH kit have been previously described [21]. Positive signals were indicated by the dark blue intranuclear staining which are indicative for presence of EBV infection in the $\mathrm{C} 17$ xenografts.

\section{Induction of lytic reactivation of EBV in the established C17 cell line}

To re-activate lytic EBV infection in the established C17 cells, the cells were transfected with a BZLF1-expressing 
plasmid p509 [22] (kindly provided by Prof. Wolfgang Hammerschmidt, German Research Center for Environmental Health, Munich, Germany) using the X-tremeGene HP DNA transfection reagent (Roche) following the manufacturer-recommended instructions. After $24 \mathrm{~h}$ of transfection, the $\mathrm{C} 17$ cells were cultured in fresh medium. The transfected cells were harvested at 24,48 , and $72 \mathrm{~h}$ after transfection and extracted for DNA, RNA, and protein to detect the EBV copy number and the expression levels of EBV latent and/or lytic genes.

\section{Assay for presence of infectious EBV particles in the supernatants of $\mathrm{C} 17$ cells after lytic reactivation by BZLF1}

After lytic reactivation of EBV infection in the established C17 cell line by expression of BZLF1, the culture supernatants from the BZLF1-transfected and untransfected C17 cells were collected. The harvested supernatants were centrifuged at $800 \mathrm{rpm}$ for $5 \mathrm{~min}$ and then filtered through a $0.45 \mu \mathrm{m}$ cellulose acetate filter to remove the cell debris. Then the centrifuged and filtered supernatants, which contain the EBV particles, were further subjected to ultracentrifugation at $20,000 \mathrm{rpm}$ for $4 \mathrm{~h}$ at $4{ }^{\circ} \mathrm{C}$ to pellet the EBV particles. The supernatant was discarded and the pellet was resuspended using RPMI 1640 medium supplemented with $10 \%$ fetal bovine serum in $1 / 30$ of the original volume. These procedures concentrated the EBV concentration in the culture supernatant by $30 \times$. EBV-negative Akata cells were then incubated with the concentrated EBV supernatant for 3 days. The infected Akata cells were harvested and subjected to DNA and RNA extraction to detect the presence of EBV copy and EBV gene expression, respectively.

\section{Results}

\section{Establishment and growth history of $\mathrm{C} 17$ cell line from the NPC xenograft}

The difficulty in establishing EBV+ve NPC cell lines that can be propagated continuously suggested that the culture conditions for NPC cells have not been optimized. The C17 was not grown immediately as a monolayer culture (Fig. 1a). The explants did not attach well to the culture surfaces but spontaneously formed tumor spheroids from the $\mathrm{C} 17$ xenografts, which could be propagated. The ability to grow as tumor spheroids may suggest that EBV-infected NPC cells prefer a three-dimensional (3D) growth condition in culture. The inclusion of Rho-associated kinase (ROCK) inhibitor (Y-27632) [7] was crucial for the growth and propagation of these NPC spheroids in culture (Fig. 1b). Tumor spheroids were formed only when ROCK inhibitor was included in culture medium. These tumor spheroids were tumorigenic when injected back into immune-deficient mice (results not shown). These 3D tumor spheroids could be expanded and propagated in culture over long periods of time ( $>500$ days). During this period, we observed continuous growth and increase in size of $\mathrm{C} 17$ spheroids. Some of the larger size tumor spheroids eventually settled down and attached onto the culture surface with epithelial outgrowths from the attached spheroids (Fig. 1c). We carefully monitored the growth conditions of the epithelial outgrowth migrating out from the attached spheroids using mixed fresh and conditioned culture medium. The epithelial cells in the outgrowth continued to proliferate and could be propagated in monolayer and passaged over 170 population doublings without any sign of senescence. The average doubling time of the $\mathrm{C} 17$ cell line is around 4.5 days. The morphology of the monolayer $\mathrm{C} 17$ cell line at population doubling (PD) 155 is shown in Fig. 1d.

\section{Short tandem repeat (STR) analysis for the authenticity of C17 cell line from its parental NPC xenograft}

Earlier reports have shown that many common NPC cell lines used in the investigation either have identical STR profiles and/or contamination of genetic elements from HeLa cells [2,3] suggestive of common origin and potential contamination from HeLa cells. We have performed short tandem repeat analysis to confirm the authenticity of this newly established EBV+ve NPC cell line, C17, from the original NPC xenograft passaged in nude mice. The genotypes at 15 STR loci (D5S818, D13S317, D7S820, D16S539, vWA, TH01, TPOX, CSF1PO, D3S1358, D21S11, D18S51, Penta E, Penta D, D8S1179, and FGA) and amelogenin at the sex chromosomes were examined and compared (Supplementary Table 2). The STR profiles of $\mathrm{C} 17$ cell line and the parental $\mathrm{C} 17$ xenograft are identical, but differ from the other NPC cell lines used in the field [2].

\section{Cytogenetic analysis of C17 cell line}

Twenty-five metaphase spreads of C17 cells were analyzed using the first passage of the cells in the epithelial outgrowth from the tumor spheroids. Spectral karyotyping showed that C17 cell line is a near-diploid cell line with stabilized structural aberrations on most of the chromosomes. Identical chromosome aberrations were detected in 21 out of 25 analyzed metaphases (Fig. 2a). All structural aberrations in the $\mathrm{C} 17$ cell line differ from those of previously reported EBV+ve C666-1 cell line [4] or other EBV-negative NPC cell lines. This further excludes the possibility of cell contamination from previous NPC cell lines. We compared the cytogenetic report published 25 years ago on short term 
Fig. 1 Growth history of $\mathrm{C} 17$ cell line. a Growth curve of $\mathrm{C} 17$ cell line. Tissues from $\mathrm{C} 17$ xenograft were cut into small pieces and seeded in culture. By including ROCK inhibitor (Y27632) in the culture medium (RPMI 1640 medium supplemented with $10 \%$ fetal bovine serum, $100 \mathrm{U} / \mathrm{ml}$ penicillin and $100 \mu \mathrm{g} / \mathrm{ml}$ streptomycin), spontaneous growth of $\mathrm{C} 17$ as tumor spheroids were observed (b) (scale bar: $500 \mu \mathrm{m})$. The $\mathrm{C} 17$ tumor spheroids grew and increased in size. The tumor spheroids increased in number by the spontaneous dissociation of spheroids and they were propagated by passing to culture flasks. Around 500 days after growth as spheroids, some of the larger $\mathrm{C} 17$ spheroids attached to the culture surfaces (c) (scale bar: $500 \mu \mathrm{m})$. Epithelial outgrowths were observed from the attached spheroids, which could be propagated as monolayer culture and finally became a continuous cell line (d) (scale bar: $100 \mu \mathrm{m})$.

a

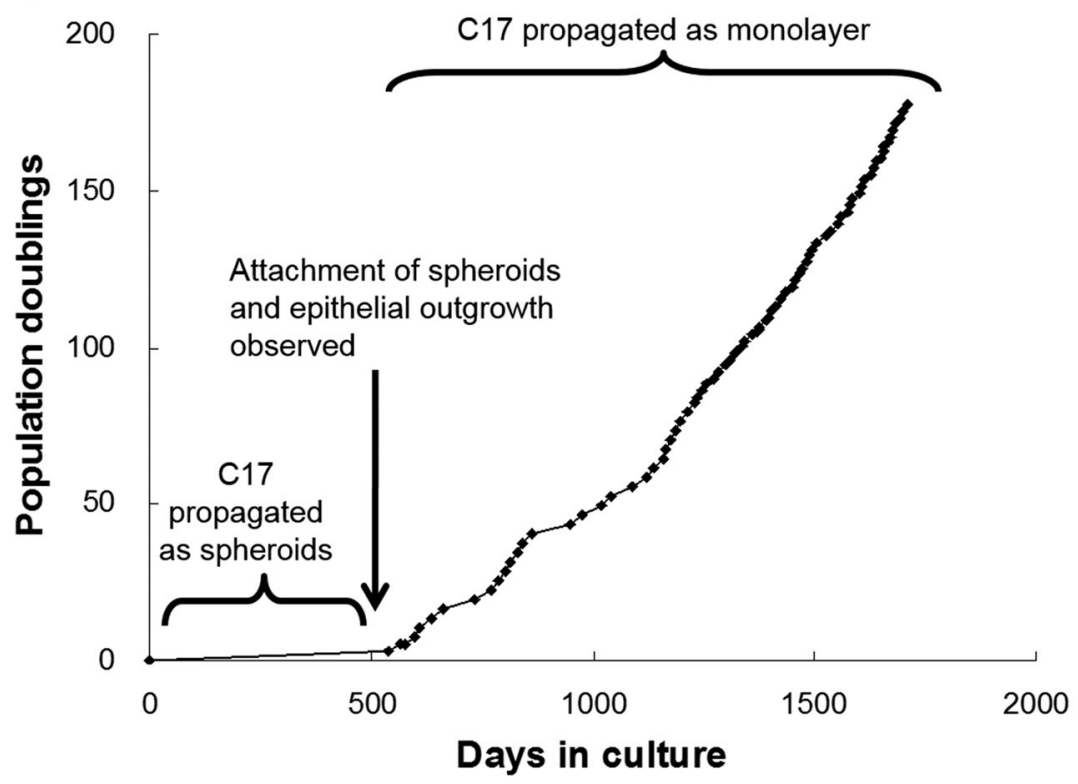

b

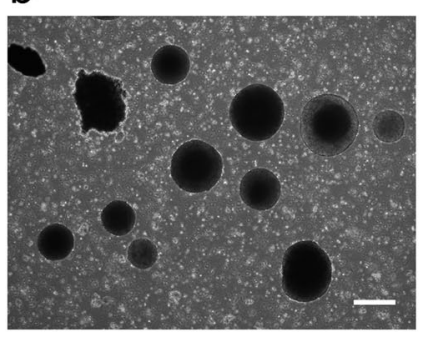

C

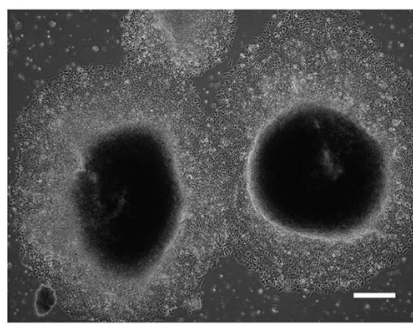

d

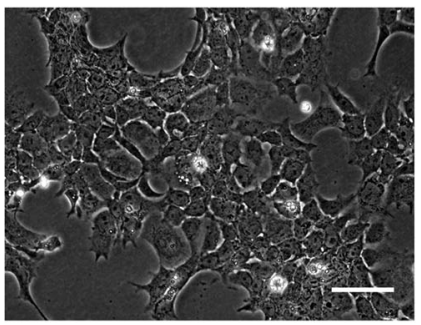

in vitro culture of $\mathrm{C} 17$ cells using G-banding techniques [23], with the cytogenetic analysis in this study using spectral karyotyping. Interestingly, some identical or similar derivative chromosomes identified in earlier study were preserved in the established $\mathrm{C} 17$ cell lines, which include the identical der(3)t $(3 ; 8)$, as well as $\operatorname{der}(6 ; 12)$ and $\operatorname{der}(5) \mathrm{t}(5 ; 9) \mathrm{t}$ $(8 ; 9)$ reported as $\operatorname{der}(12) \mathrm{t}(6 ; 12)$ and $\operatorname{der}(5) \mathrm{t}(5 ; 8)$, respectively, in the earlier report. The use of spectral karyotyping has identified additional derivative chromosomes not described in the earlier study. The molecular identity of the genes involved remained to be elucidated.

\section{Anchorage-independent growth and tumorigenicity of C17 cell line}

The anchorage-independent growth is a good indicator of in vitro transformation property of cancer cell lines. Soft agar colony formation assay was performed in the established C17 cell line at PD 79. Results showed that the C17 cells could undergo anchorage-independent growth with 77 \pm 0.71 colonies detected in $5 \times 10^{4}$ cells seeded as single cells in soft agar. A representative image of the soft agar colonies is shown in Fig. 2 b.
The in vivo tumorigenic property of the established cell line (at PD 76) was further confirmed in immune-deficient animals. Six NOD/SCID mice were injected subcutaneously with $10^{7} \mathrm{C} 17$ cells mixed in 1:1 ratio of Matrigel and culture medium in a total of $200 \mu \mathrm{l}$. Tumorigenic growth was observed in six out of six mice injected (Fig. 2c). The tumor size was around $2 \mathrm{~cm}^{3}$ at day 34 after injection. These results confirmed the established $\mathrm{C} 17$ cells retained the tumorigenic property of the parental C17 xenograft. Figure $2 \mathrm{~d}$ shows the representative image and appearance of the tumor formed from injection of $\mathrm{C} 17$ in NOD/SCID mice. Histological sections revealed similar undifferentiating histological features in the tumor from the established C17 cell line and the parental C17 tumor xenografts (Fig. 2e). In situ hybridization of EBER also confirmed the presence of EBV in tumors from the newly established C17 cell line and the parental C17 xenograft.

\section{EBV infection status in C17 cell line}

The EBER ISH study confirmed the presence of EBV in the tumor xenografts derived from the established $\mathrm{C} 17$ cell line. We further confirmed the presence of EBV infection in the 
a

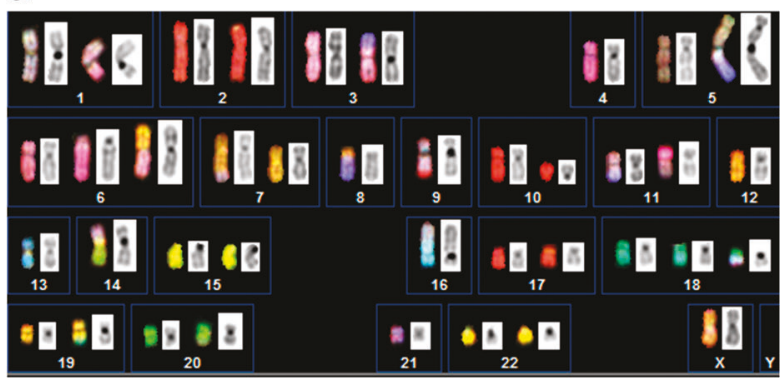

b

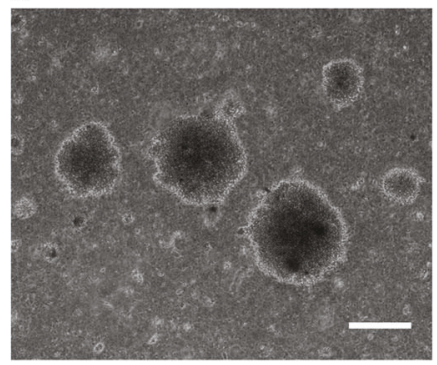

C

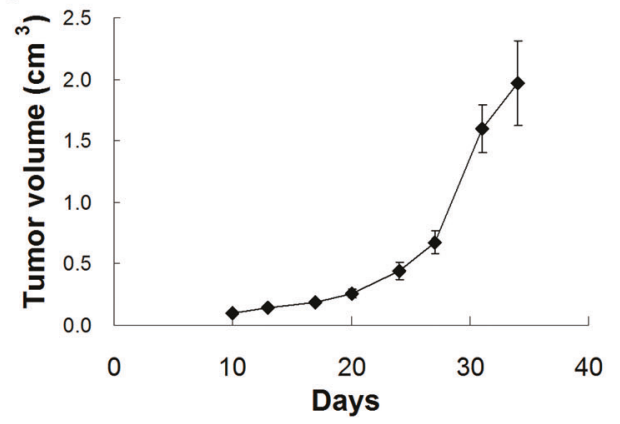

d

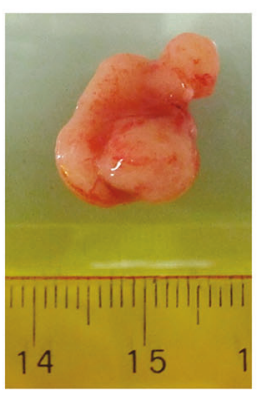

e

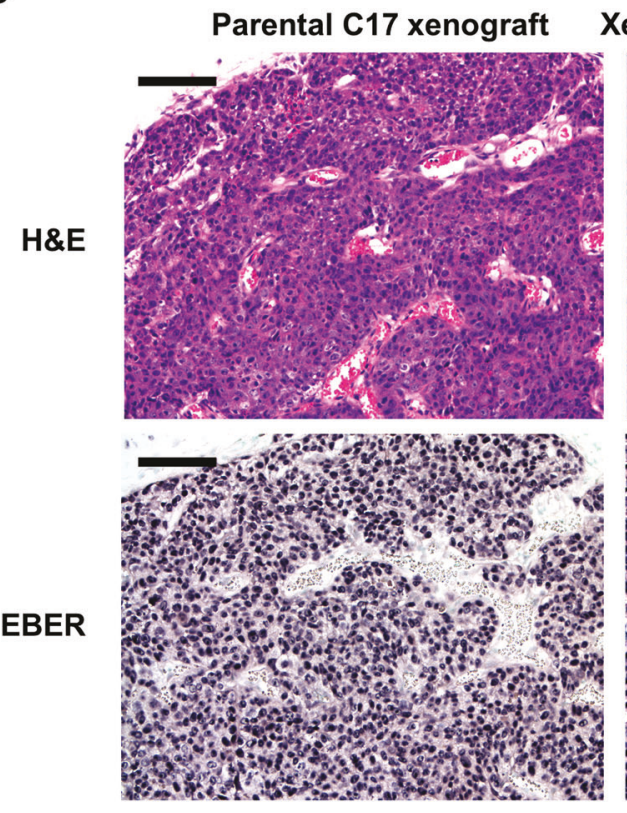

Xenograft generated from $\mathrm{C} 17$ cell line
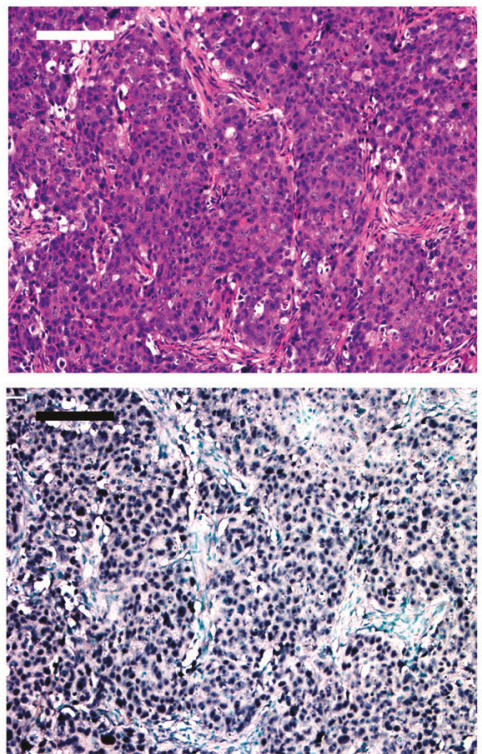

newly established $\mathrm{C} 17$ cell line grown as monolayer in culture and as tumor xenograft in NOD/SCID mice by realtime PCR for the presence of EBV DNA. Results showed that the EBV DNA, EBNA1, could be detected in the similar passage (PD 76-90) in both the $\mathrm{C} 17$ cell line propagated as monolayer in vitro and as tumor xenograft in vivo (Fig. 3a). Interestingly, we detected a higher EBV copy in $\mathrm{C} 17$ cells grown as tumor xenograft $(8.12 \pm 0.74$ per cell) compared to $\mathrm{C} 17$ cells grown as monolayer in culture (3.17 \pm 0.09 per cell). This may suggest that EBV infection may provide selective growth advantage to $\mathrm{C} 17$ growth in 3D culture condition, which mimics more the growth of NPC cells in vivo. This observation is consistent with the finding of the universal presence of EBV infection in almost all the undifferentiated NPCs in patients and in all the established NPC xenografts passaged in immune-deficient 
Fig. 2 Characteristics of $\mathrm{C} 17$ cell line. a Spectral karyotyping of $\mathrm{C} 17$ cell line. The karyotype of $\mathrm{C} 17$ is as follows: 39,X,-Y?,del(1)(p3?),der (1)t(1;?3)(q21;?),der(2)t(2;?12)(p24;?),der(3)t(3;8)(p24;q21),-4,dup $(?)(5)(\mathrm{q} ?), \operatorname{der}(5) \mathrm{t}(5 ; 9)(\mathrm{q} 31 ; \mathrm{q} 11) \mathrm{t}(8 ; 9)(\mathrm{q} 11 ; \mathrm{q} 2 ?),+6, \operatorname{der}(6) \operatorname{del}(6)(\mathrm{p} 21) \mathrm{t}$ $(4 ; 6)(\mathrm{q} 12 ; \mathrm{q} 12), \operatorname{der}(6 ; 12)(\mathrm{q} 10 ; \mathrm{q} 10), \operatorname{der}(7) \mathrm{t}(7 ; 9)(\mathrm{q} 3 ? ; ?),-8, \operatorname{der}(8 ; 12)$ (q10;p10), der(9)t(2;9)(?;q3?), del(10)(q11),der(11;21)(q10;q10), der (11)t( $8 ; 11)(? ; \mathrm{q} 12), \operatorname{der}(13 ; 13)(\mathrm{q} 10 ; \mathrm{q} 10) \operatorname{del}(\mathrm{q} 31),-14, \operatorname{der}(14) \mathrm{t}(1 ; 14)$ $(\mathrm{p} 11 ; ?) \operatorname{del}(1)(\mathrm{q} ?),-16, \operatorname{der}(16) \mathrm{t}(1 ; 16)(? ; \mathrm{p} 13), \operatorname{der}(17) \mathrm{t}(12 ; 17)(\mathrm{p} 11 ; \mathrm{p} 11)$, $+18, \operatorname{der}(18) \mathrm{t}(16 ; 18)(? ; \mathrm{q} 11) \mathrm{t}(16 ; 21)(? ; ?), \operatorname{der}(19) \mathrm{t}(16 ; 19)(? ; \mathrm{q} 13), \mathrm{der}$ (20)t(5;20)(?; 13),der(21)t(21;21)(q10;q10)t(5;21)(?;q21) [21]. This karyotype reveals that $\mathrm{C} 17$ is near-diploid. Identical chromosome aberrations were detected in 21 out of 25 analyzed metaphases, indicating that this cell line retains some degree of genetic stability. b Anchorage-independent growth of $\mathrm{C} 17$ cell line as soft agar clones. Soft agar colony formation assay of $\mathrm{C} 17$ cell line showed that $77 \pm$ 0.71 colonies can be formed from $5 \times 10^{4}$ cells. Representative image of the soft agar colonies is shown (scale bar: $500 \mu \mathrm{m}$ ). c Growth of xenograft in NOD/SCID mice from injection of C17 cell line. Ten million C17 cells were mixed with Matrigel and injected subcutaneously into NOD/SCID mouse for the measurement of growth rate of xenograft. Six mice were examined. The tumor growth was measured by the formula: width $\times$ width $\times$ length $/ 2$. The tumors were measured every 3 or 4 days until the tumor was about $2 \mathrm{~cm}^{3}$. The mice were killed and tumors were harvested. d Representative image of the tumor formed. e Histology of $\mathrm{C} 17$ tumor. H\&E staining of parental C17 xenograft, and tumor xenograft formed by injection of $\mathrm{C} 17$ cells. Both xenografts are EBV-positive, as indicated by the dark blue intranuclear staining by EBER ISH. Scale bar: $100 \mu \mathrm{m}$.

mice (xeno-666, xeno-2117, C15, C18, and C17), supporting a selective growth advantage of EBV-infected NPC cells in vivo. The nature of this selective growth advantage remains elusive. Elucidation of this growth advantage of EBV-infected NPC cells in vivo may provide insights into the contribution of EBV infection to growth of NPC cells in patients. In contrast, EBV episomes are readily lost in NPC cells propagated in culture using conventional media, suggesting that EBV infection per se may not confer a growth advantage to NPC cells in monolayer culture. In the presence of ROCK inhibitor, the EBV episomes remain stable in the newly established $\mathrm{C} 17$ cell line upon propagation. The inclusion of ROCK inhibitor may influence differentiation properties of the NPC cells and contribute to the stable maintenance of EBV in the established $\mathrm{C} 17$ cell line. The long passage history of $\mathrm{C} 17$ xenograft in immune-deficient mice may also select for stable EBV-infected clones of $\mathrm{C} 17$ cells. The presence of $\mathrm{EBV}$ in the $\mathrm{C} 17$ cell line was further confirmed by interphase fluorescence in situ hybridization (FISH) for the presence of EBV genome (Fig. 3b). All the 200 cells analyzed at PD 167 were EBV+ve. The average number of EBV copies per $\mathrm{C} 17$ cell cultivated in vitro analyzed using FISH at PD 167 was $3.0 \pm 1.8$, which is comparable with the EBV copies $(3.17 \pm 0.09$ per cells) using PCR quantitation of EBV DNA at PD 90. We did not observe statistically significant drift in copy numbers per $\mathrm{C} 17$ cell at PD 167, when compared with that at earlier population doublings 5 and $100(p>0.05)$.
We then determined the EBV gene expression profiles in the $\mathrm{C} 17$ cell line. Both the $\mathrm{C} 17$ cell line and xenograft showed a similar expression level of EBV genes. Expression of latent transcripts of EBV genes (EBNA1, EBERI/2, $L M P 1$, and $L M P 2$ ) could be detected in the newly established $\mathrm{C} 17$ cell line (Fig. 3c). The expression of EBV lytic genes (BZLF1, BRLF1, BMRF1, and BLLF1) was low in C17 cells compared to C666-1. The profiles of EBV gene expression detected in $\mathrm{C} 17$ cells are representative of type II latent $\mathrm{EBV}$ infection, which is characteristic of latent $\mathrm{EBV}$ infection in NPC.

\section{Reactivation of lytic EBV infection in C17 cell line}

We then examined if lytic infection of EBV could be induced in the newly established monolayer culture of $\mathrm{C} 17$ NPC cells (at PD 106-169). The BZLF1, which is the immediate early transactivator initiating the switching of latent to lytic infection of EBV in infected cells, was expressed by transient transfection in the $\mathrm{C} 17$ cell line. The successful expression of BZLF1 in C17 cells at PD 117 was confirmed by the increased expression of $B Z L F 1$ transcripts in transfected C17 cells by RT-PCR (Fig. 4a). Increased expression of other EBV lytic transcripts, BRLF1, BMRF1, and $B L L F 1$, were also detected following expression of $B Z L F 1$. The BRLF1 is an immediate early transactivator activated by BZLF1, which plays an important role in lytic reactivation of EBV in infected epithelial cells [24]. The $B M R F 1$ is an early EBV gene that encodes the polymerase accessory protein (EA-D), which is essential for lytic replication of EBV DNA. The $B L L F 1$ is the late EBV gene encoding the major viral glycoproteins, gp350/220, which are involved in EBV infection. The increased expression of immediate, early, and late lytic EBV genes suggested productive lytic EBV reactivation in $\mathrm{C} 17$ cells. Expression of EBV latent genes, EBNA1, EBER1/2, LMP1, and $L M P 2$, were detected in the $\mathrm{C} 17$ cells, but remained fairly unchanged in expression levels, except for LMP1. Expression of LMP1 increased significantly following the expression of BZLF1. The increased expression of LMP1 conformed to recent reports that LMP1 expression may play an important role in lytic infection of $\operatorname{EBV}[25,26]$.

The increase in protein expression of BZLF1 (Zta), BRLF1 (Rta), and BMRF1 (EA-D) was also confirmed in the BZLF1-transfected C17 cells (Fig. 4b). Expression of Zta was detected in the $\mathrm{C} 17$ cells $24 \mathrm{~h}$ after BZLF1 transfection. Expression of Rta, which is encoded by the BRLF1 gene, was detected only after expression of Zta at $48 \mathrm{~h}$ time point and continued to increase at $72 \mathrm{~h}$ time point after BZLF1 transfection. The EA-D protein was detectable after the expression of the two immediate early proteins (Zta and Rta) and was detected at a higher level at $72 \mathrm{~h}$ time point after BZLF1 transfection. The copy number of EBV DNA 
Fig. 3 EBV infection status of C17 cell line. a EBV DNA copy (mean $\pm \mathrm{SD}$ ) could be detected in both $\mathrm{C} 17$ xenograft and cell line by quantitative PCR. b Typical interphase EBV FISH at population doubling 167 showed the presence of EBV in $\mathrm{C} 17$ cell line as indicated by the bright red dots in nucleus. c EBV gene expression profile by quantitative RT-PCR. C17 cell line expressed the latent EBV genes (EBNA1, EBER1/2, $L M P 1$, and $L M P 2$ ), which are characteristics of type II EBV latency. The lytic genes (BZLF1, $B R L F 1, B M R F 1$, and $B L L F 1)$ were expressed at a lower level in the $\mathrm{C} 17$ cell line compared to C666-1 cells. a

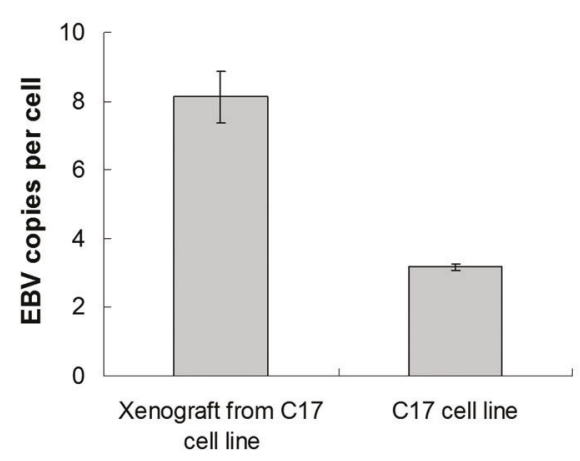

C

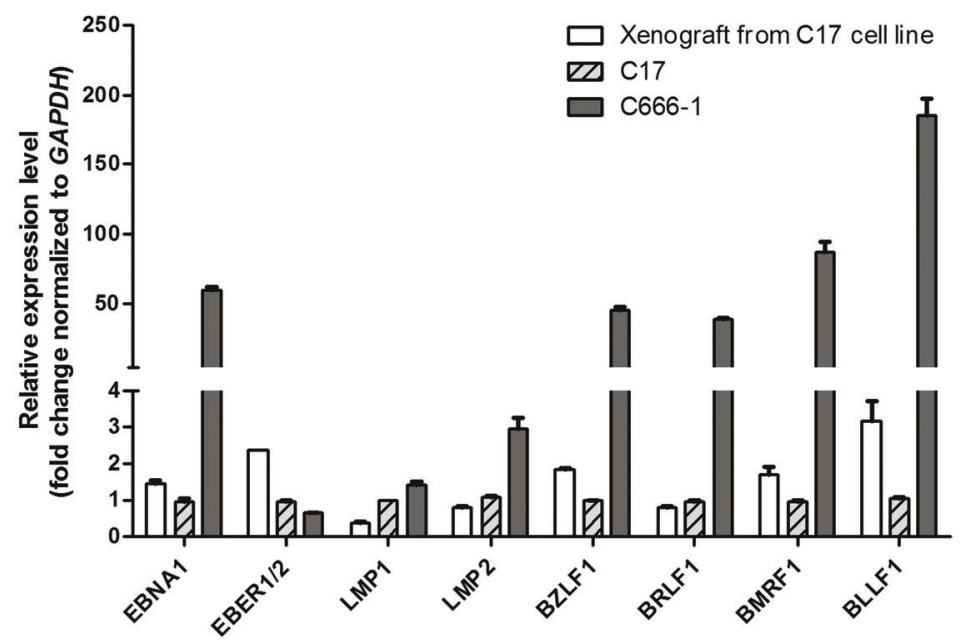

was also significantly increased by more than fourfold in C17 cells transfected with BZLF1 compared to untransfected $\mathrm{C} 17$ cells (Fig. 4c). The increased transcription levels of lytic genes and proteins of EBV, as well as EBV DNA copy in $\mathrm{C} 17$ cells upon transfection of BZLF1, support the activation of lytic infection of EBV in $\mathrm{C} 17$ cells.

We then examined if infectious EBV particles could be detected in the supernatant harvested from $\mathrm{C} 17$ cells transfected with BZLF1. Supernatants from BZLF1-transfected and untransfected C17 cells were harvested and centrifuged at high speed to concentrate the EBV particles, if they were present in the supernatants. The concentrated supernatant was then used to infect the EBV-negative Akata cells to detect the expression of EBV genes and EBV DNA in the infected cells. The procedures are shown in the schematic diagram in Fig. 4d. Results showed that the EBV DNA was increased by 175 -fold in the Akata cells incubated with supernatant harvested from BZLF1-transfected C17 cells compared to Akata cells incubated with supernatant harvested from control untransfected $\mathrm{C} 17$ cells (Fig. 4e). Expression of EBV genes could also be detected in Akata cells incubated with concentrated supernatant b

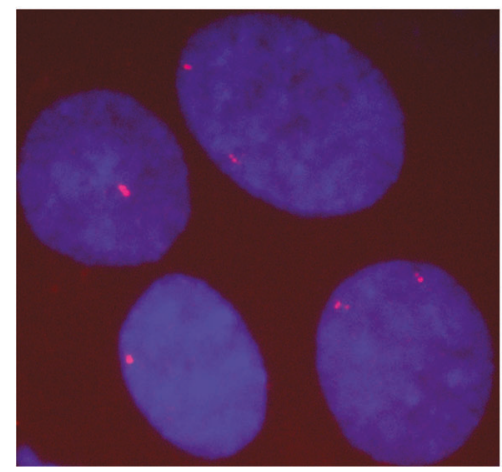

harvested from BZLF1-transfected C17 cells, but not from control untransfected $\mathrm{C} 17$ cells (Fig. 4f). All these results clearly demonstrated that productive EBV lytic infection could be induced in $\mathrm{C} 17$ cell line after expression of BZLF1.

\section{Discussion}

The difficulty in establishment of representative NPC cell lines harboring EBV has seriously hampered research and investigation on EBV infection in NPC. At present, there is only one NPC cell line, C666-1, which harbors EBV. The lack of representative NPC cell lines also limited preclinical testing of novel and effective therapeutic agents for treating NPC. The underlying reasons for the difficulty in establishment of NPC cells are not clearly understood. The limited access and small sizes of NPC biopsies may contribute to the lack of success in establishment of NPC cell lines. In addition, the growth conditions that are successful in establishment of head and neck cancer cell lines apparently are suboptimal for establishment of NPC cells as 

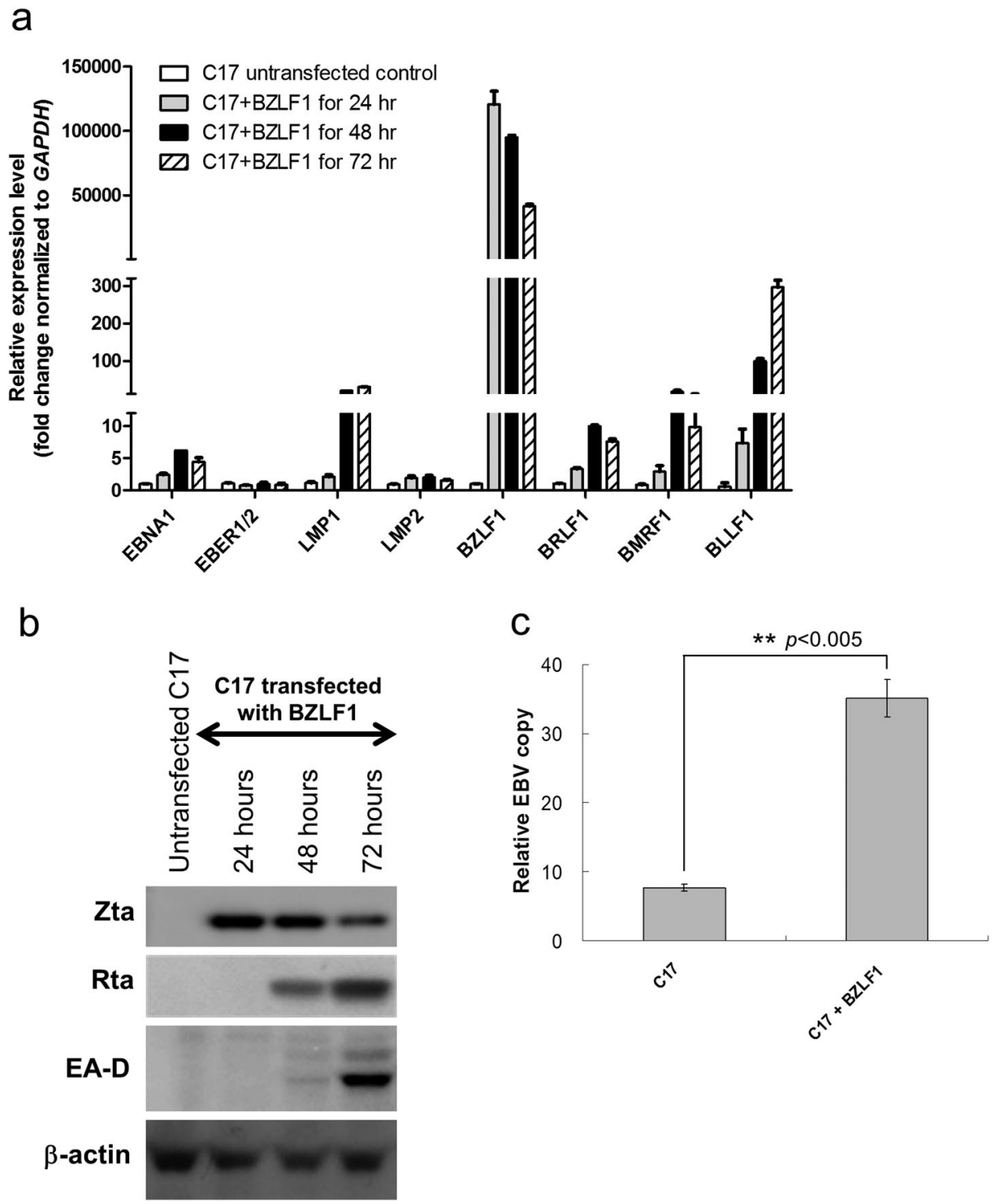

Fig. 4 (Continued on next page)

shown in the low successful rate of establishment of NPC cell lines. NPC differs from other head and neck cancer in its undifferentiated properties and the presence of EBV infection. Another distinct feature of NPC is the heavy infiltration of lymphoid and inflammatory components, which may release cytokines to support growth of NPC cells in patients and may also be involved in the persistence of EBV infection in NPC. Defining the role of these lymphoid and stromal cells in the tumor microenvironment of NPC is essential as it will contribute to the understanding of growth requirement of NPC cell lines and the role of EBV in its pathogenesis.

EBV infection has been postulated to play an important role in NPC development and progression in patients. Counterintuitively, the presence of EBV infection in NPC may contribute to the difficulty in establishment of NPC cell lines in culture. Our postulation is that the unique microenvironment of NPC plays essential roles in regulating the balance of latent and lytic EBV infection. The absence of these supportive tumor microenvironments in NPC cells when they are explanted to culture may trigger lytic EBV reactivation, which induces growth arrest in EBV-infected NPC cells and possibly leads to their failure to be established in culture. This postulation also conforms to the observation that all NPC cell lines established in culture, except C666-1 which is defective in lytic reactivation of EBV, rapidly lost their EBV episomes upon propagation. The abortive nature of lytic activation of EBV in C666-1 cells may enable the cells to retain the infected EBV episomes upon propagation. 


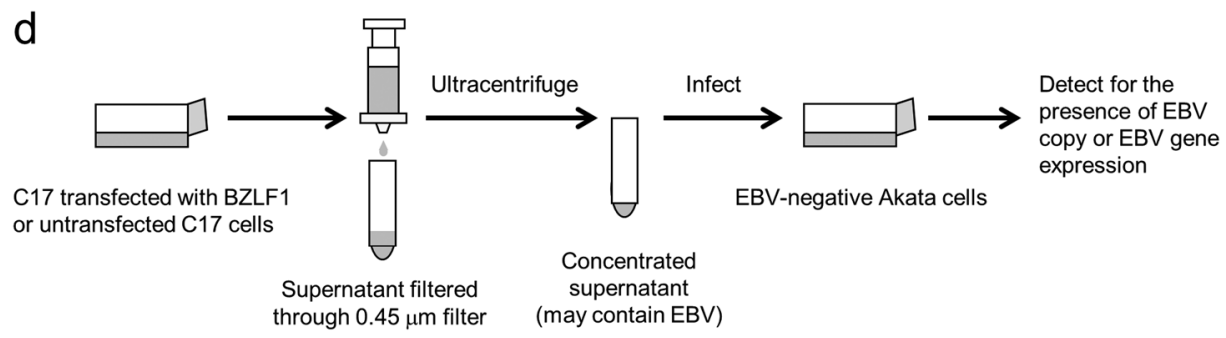

e

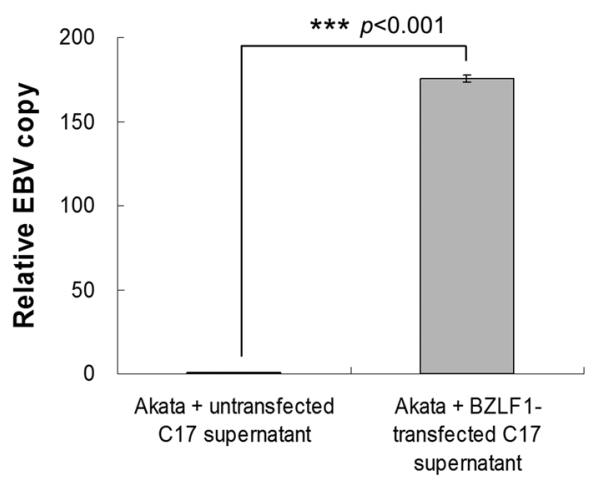

$f$

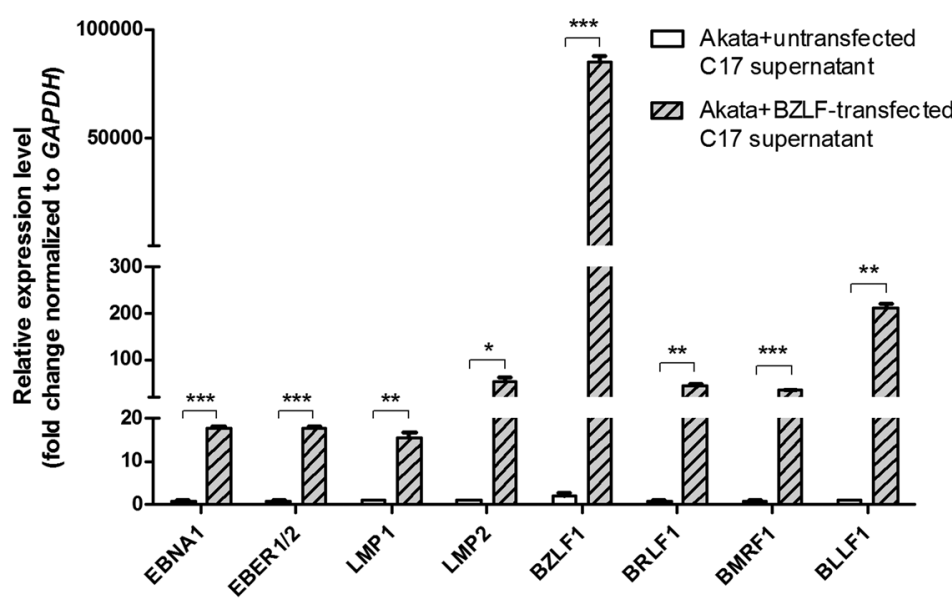

Fig. 4 (Continued) Reactivation of EBV lytic cycle in C17 cell line. a EBV gene expression profile of $\mathrm{C} 17$ cells upon transfection of BZLF1. Transfection of BZLF1 to $\mathrm{C} 17$ cells induced the expression of other EBV lytic genes including $B R L F 1, B M R F 1$, and $B L L F 1$, indicative of induction of lytic reactivation of EBV in infected cells. b After the expression of Zta (protein encoded by BZLF1), the expression of other EBV lytic proteins (Rta and EA-D) were also increased. $\mathbf{c}$ The relative EBV copy significantly increased in $\mathrm{C} 17$ cells after expression of BZLF1. Unpaired $t$ test, $p<0.005$. d Schematic diagram for the procedures to assay for the presence of infectious EBV particles in supernatant harvested from $\mathrm{C} 17$ cells induced to undergo lytic reactivation. Supernatants were collected from the $\mathrm{C} 17$ cells transfected with BZLF1 or from the control untransfected $\mathrm{C} 17$ cells. The supernatants were briefly centrifuged and filtered through a $0.45 \mu \mathrm{m}$ cellulose acetate filter to remove the cell debris. Then the centrifuged and

A previous study has already shown that cell line establishment from xenografted tissues represents a more efficient alternative compared to fresh tumor specimens [5]. filtered supernatants containing the EBV particles were further subjected to ultracentrifugation to pellet the EBV particles. The pellet was resuspended in medium of 1/30 of the original volume. EBV-negative Akata cells were then incubated with the concentrated EBV for 3 days. The infected Akata cells were harvested and subjected to DNA and RNA extraction to detect the presence of EBV copy and EBV gene expression, respectively. e EBV infection assay showed that the EBVnegative Akata cells infected with the supernatant from BZLF1transfected $\mathrm{C} 17$ cells expressed a significantly higher EBV copy than those infected with supernatant from untransfected $\mathrm{C} 17$ cells. Unpaired $t$ test, $p<0.001$. f EBV infection assay also revealed that the EBVnegative Akata cells infected with the supernatant from BZLF1transfected $\mathrm{C} 17$ cells expressed the EBV genes at a significantly higher level than those infected with supernatant from untransfected $\mathrm{C} 17$ cells. Unpaired $t$ test, $* p<0.05, * * p<0.005$, and $* * * p<0.001$.

In general, human tumor xenografts that have been passaged in immune suppressed animals are commonly enriched in their tumor cell content and decreased in stromal 
content. The tumor cells grown as transplanted xenografts may be less dependent on the original tumor microenvironment in patients for growth. The NPC microenvironment in patients may be essential to suppress lytic infection and support latent EBV infection. Expression of EBV latent genes, such as the BART-microRNA, which is highly expressed in NPC, has been shown to suppress apoptosis in NPC cells [27]. The NPC xenografts may be valuable resources for future establishment of NPC cell lines. In addition to $\mathrm{C} 17$ xenografts, there are $\mathrm{C} 15$ and $\mathrm{C} 18$ xenografts established from NPC patients from North Africa and France [13], respectively, and xeno-666 and xeno-2117 from Southern Chinese [28]. Using conventional culture methods, we were able to establish the C666-1 from NPC xenograft, xeno-666, but failed to establish cell lines from other NPC xenografts. Epithelial outgrowths were observed in some of these NPC xenografts (e.g., xeno2117), but they failed to proliferate and were eventually lost from the culture (unpublished observations). The use of tumor spheroid culture and inclusion of ROCK inhibitor (Y27632) in the culture medium have contributed to the successful establishment of C17. Using similar approaches, more $\mathrm{EBV}+\mathrm{ve}$ NPC cell lines may be established from xenografted NPC in future.

In this study, the initial growth of NPC cells as tumor spheroids may allow adaptation of NPC cells to grow in culture. Tumor spheroids from $\mathrm{C} 17$ were propagated for over 500 days before their attachment to culture surfaces which initiated monolayer growth. The use of ROCK inhibitor facilitated the formation and growth of tumor spheroids as reported in primary colon cancer [29]. Spheroid culture is commonly used in stem cell culture. The growth of $\mathrm{C} 17$ as spheroids may support the growth of undifferentiated NPC cells with enhanced stem cell-like properties. Our preliminary observation showed that spheroid culture represents a more favorable growth condition over monolayer cell culture for NPC cells. The 3D culture conditions, which facilitate cell-cell interaction, may be essential for survival of NPC cells, and may suppress lytic EBV reactivation and the lyticinduced growth arrest in infected NPC cells which interferes with cell line establishment.

Recent studies have shown that ROCK inhibitor (Y-27632) could prevent onset of growth arrest, inhibit terminal differentiation, but increase cell proliferation and immortalize keratinocytes [9-11]. ROCK inhibitor is commonly used in the growth of stem cells isolated from tissues $[14,30]$. The detailed mechanisms involved in regulation of differentiation and stemness in cells by Y-27632 remains to be elucidated. Nonetheless, we speculate that the suppression of differentiation [12] and maintenance of stemness $[8,22]$ by ROCK inhibitor may be two supportive factors for growth of NPC cells in vitro, leading to the successful establishment of the $\mathrm{C} 17$ cell line. Undifferentiated NPC is the predominant histopathological type of NPC in the endemic regions. The presence of EBV is universally associated with undifferentiated NPC, suggesting that intracellular signaling environment in undifferentiated NPC may be supportive for latent EBV infection. Latent EBV infection is an essential step in NPC pathogenesis. EBV, however, is rapidly lost in NPC cell lines upon prolonged propagation [31-33]. It may reflect the change of differentiation properties of NPC cells under suboptimal culture conditions, which induce lytic reactivation of EBV to suppress growth of EBV-infected NPC cells.

The presence of the ROCK inhibitor, which suppresses the differentiation properties of NPC cells grown in culture, may contribute to the persistence of EBV infection in the newly established $\mathrm{C} 17$ cell line by suppressing lytic reactivation of EBV in NPC cells. Viral replication may cause DNA damage in host cells and trigger apoptosis [24, 27, 34], which may be an explanation for the rapid loss of episomal viral genomes from cultured epithelial cells. ROCK inhibitor reverses blebbing of keratinocytes and inhibits apoptotic pathways [35]. This might be another reason why inclusion of ROCK inhibitor leads to establishment of EBVcontaining NPC cell lines.

The C17 cell line has undergone over 170 population doublings and showed no sign of senescence. The persistence of EBV in $\mathrm{C} 17$ cells was confirmed by the detection of EBV DNA, EBER hybridization, interphase EBV DNA FISH, expression of EBV latent and lytic genes, and the generation of infectious EBV upon lytic reactivation by expression of BZLF1. In addition to its use in preclinical drug evaluation for NPC treatment, the $\mathrm{C} 17$ cell line may also have applications in the study of regulation of latent and lytic EBV infection in infected NPC cells. The C17 cell line represents a bona fide NPC-derived cell line harboring the original EBV presents in the NPC patient. The establishment of the $\mathrm{C} 17$ cell line and its susceptibility to undergo lytic reactivation also opens up the opportunity for cloning of additional NPC-derived EBV after the M81 EBV [36], which has distinct infection properties, and to address the important question whether distinct EBV strains may be associated with human cancers.

Acknowledgements We acknowledge the generous funding sources for the above study. HMRF grant (04151726) and funding from Hong Kong Research Grant Council: general research fund (HKU 779713M), AoE NPC grant (AoE/M-06/08), Theme-based research scheme grant (T12-401/13-R) and collaborative research fund (C702716G and C1013-15G).

\section{Compliance with ethical standards}

Conflict of interest The authors declare that they have no conflict of interest. 


\section{References}

1. Lo KW, To KF, Huang DP. Focus on nasopharyngeal carcinoma. Cancer Cell. 2004;5:423-8.

2. Chan SY, Choy KW, Tsao SW, et al. Authentication of nasopharyngeal carcinoma tumor lines. Int J Cancer. 2008;122:2169-71.

3. Strong MJ, Baddoo M, Nanbo A, et al. Comprehensive highthroughput RNA sequencing analysis reveals contamination of multiple nasopharyngeal carcinoma cell lines with HeLa cell genomes. J Virol. 2014;88:10696-704.

4. Cheung ST, Huang DP, Hui AB, et al. Nasopharyngeal carcinoma cell line (C666-1) consistently harbouring Epstein-Barr virus. Int J Cancer. 1999;83:121-6.

5. Dangles-Marie V, Pocard M, Richon S, et al. Establishment of human colon cancer cell lines from fresh tumors versus xenografts: comparison of success rate and cell line features. Cancer Res. 2007;67:398-407.

6. Watanabe K, Ueno M, Kamiya D, et al. A ROCK inhibitor permits survival of dissociated human embryonic stem cells. Nat Biotechnol. 2007;25:681-6.

7. Liu X, Ory V, Chapman S, et al. ROCK inhibitor and feeder cells induce the conditional reprogramming of epithelial cells. Am J Pathol. 2012;180:599-607.

8. Liu X, Krawczyk E, Suprynowicz FA, et al. Conditional reprogramming and long-term expansion of normal and tumor cells from human biospecimens. Nat Protoc. 2017;12:439-51.

9. Verillaud B, Gressette M, Morel Y, et al. Toll-like receptor 3 in Epstein-Barr virus-associated nasopharyngeal carcinomas: consistent expression and cytotoxic effects of its synthetic ligand poly (A:U) combined to a Smac-mimetic. Infect Agent Cancer. 2012;7:36.

10. McMullan R, Lax S, Robertson VH, et al. Keratinocyte differentiation is regulated by the Rho and ROCK signaling pathway. Curr Biol. 2003;13:2185-9.

11. Chapman S, Liu X, Meyers C, et al. Human keratinocytes are efficiently immortalized by a Rho kinase inhibitor. J Clin Invest. 2010;120:2619-26.

12. Palechor-Ceron N, Suprynowicz FA, Upadhyay G, et al. Radiation induces diffusible feeder cell factor(s) that cooperate with ROCK inhibitor to conditionally reprogram and immortalize epithelial cells. Am J Pathol. 2013;183:1862-70.

13. Busson P, Ganem G, Flores P, et al. Establishment and characterization of three transplantable EBV-containing nasopharyngeal carcinomas. Int J Cancer. 1988;42:599-606.

14. Gressette M, Verillaud B, Jimenez-Pailhes AS, et al. Treatment of nasopharyngeal carcinoma cells with the histone-deacetylase inhibitor abexinostat: cooperative effects with cis-platin and radiotherapy on patient-derived xenografts. PLoS ONE. 2014;9: e91325.

15. Deng W, Tsao SW, Lucas JN, et al. A new method for improving metaphase chromosome spreading. Cytom A. 2003;51:46-51.

16. Goessel G, Quante M, Hahn WC, et al. Creating oral squamous cancer cells: a cellular model of oral-esophageal carcinogenesis. Proc Natl Acad Sci USA. 2005;102:15599-604.

17. Lawrence JB, Villnave CA, Singer RH. Sensitive, high-resolution chromatin and chromosome mapping in situ: presence and orientation of two closely integrated copies of EBV in a lymphoma line. Cell. 1988;52:51-61.

18. Yip YL, Tsao SW. Regulation of p63 expression in primary and immortalized nasopharyngeal epithelial cells. Int $\mathrm{J}$ Oncol. 2008;33:713-24.
19. Lo AK, Huang DP, Lo KW, et al. Phenotypic alterations induced by the Hong Kong-prevalent Epstein-Barr virus-encoded LMP1 variant (2117-LMP1) in nasopharyngeal epithelial cells. Int J Cancer. 2004;109:919-25.

20. Tsang CM, Yip YL, Lo KW, et al. Cyclin D1 overexpression supports stable EBV infection in nasopharyngeal epithelial cells. Proc Natl Acad Sci USA. 2012;109:E3473-3482.

21. Pak MW, To KF, Lo YM, et al. Nasopharyngeal carcinoma in situ (NPCIS)--pathologic and clinical perspectives. Head Neck. 2002;24:989-95.

22. Suprynowicz FA, Upadhyay G, Krawczyk E, et al. Conditionally reprogrammed cells represent a stem-like state of adult epithelial cells. Proc Natl Acad Sci USA. 2012;109:20035-40.

23. Bernheim A, Rousselet G, Massaad L, et al. Cytogenetic studies in three xenografted nasopharyngeal carcinomas. Cancer Genet Cytogenet. 1993;66:11-15.

24. Wasil LR, Wei L, Chang C, et al. Regulation of DNA damage signaling and cell death responses by Epstein-Barr virus latent membrane protein 1 (LMP1) and LMP2A in nasopharyngeal carcinoma cells. J Virol. 2015;89:7612-24.

25. Ahsan N, Kanda T, Nagashima K, et al. Epstein-Barr virus transforming protein LMP1 plays a critical role in virus production. J Virol. 2005;79:4415-24.

26. Nawandar DM, Ohashi M, Djavadian R, et al. Differentiationdependent LMP1 expression is required for efficient lytic EpsteinBarr virus reactivation in epithelial cells. J Virol. 2017;91: e02438-16.

27. Sinclair A, Yarranton S, Schelcher C. DNA-damage response pathways triggered by viral replication. Expert Rev Mol Med. 2006;8:1-11.

28. Huang DP, Lau WH, Lung M, et al. Establishment and characterization of undifferentiated nasopharyngeal carcinoma xenografts from southern Chinese. J Exp Clin Cancer Res. 1988;7:48.

29. Ohata H, Ishiguro T, Aihara $\mathrm{Y}$, et al. Induction of the stem-like cell regulator $\mathrm{CD} 44$ by Rho kinase inhibition contributes to the maintenance of colon cancer-initiating cells. Cancer Res. 2012;72:5101-10.

30. Drost J, Karthaus WR, Gao D, et al. Organoid culture systems for prostate epithelial and cancer tissue. Nat Protoc. 2016;11: 347-58.

31. Huang DP, Ho JH, Poon YF, et al. Establishment of a cell line (NPC/HK1) from a differentiated squamous carcinoma of the nasopharynx. Int J Cancer. 1980;26:127-32.

32. Yao KT, Zhang HY, Zhu HC, et al. Establishment and characterization of two epithelial tumor cell lines (HNE-1 and HONE1) latently infected with Epstein-Barr virus and derived from nasopharyngeal carcinomas. Int J Cancer. 1990;45: 83-89.

33. Lin CT, Chan WY, Chen W, et al. Characterization of seven newly established nasopharyngeal carcinoma cell lines. Lab Invest. 1993;68:716-27.

34. Li R, Hayward SD. The Ying-Yang of the virus-host interaction: control of the DNA damage response. Future Microbiol. 2011;6:379-83.

35. Dakic A, DiVito K, Fang S, et al. ROCK inhibitor reduces Mycinduced apoptosis and mediates immortalization of human keratinocytes. Oncotarget. 2016;7:66740-53.

36. Tsai MH, Raykova A, Klinke O, et al. Spontaneous lytic replication and epitheliotropism define an Epstein-Barr virus strain found in carcinomas. Cell Rep. 2013;5:458-70. 